\title{
Epidemiology and prognosis of anti- infective therapy in the ICU setting during acute pancreatitis: a cohort study
}

Philippe Montravers ${ }^{1,2,3^{*}}$ (D), Elie Kantor ${ }^{1,2}$, Jean-Michel Constantin ${ }^{4,5}$, Jean-Yves Lefrant ${ }^{6}$, Thomas Lescot ${ }^{7}$, Nicolas Nesseler ${ }^{8}$, Catherine Paugam ${ }^{2,9}$, Matthieu Jabaudon ${ }^{4,5}$ and Hervé Dupont ${ }^{10}$

\begin{abstract}
Background: Recent international guidelines for acute pancreatitis (AP) recommend limiting anti-infective therapy (AIT) to cases of suspected necrotizing AP or nosocomial extrapancreatic infection. Limited data are available concerning empirical and documented AIT prescribing practices in patients admitted to the intensive care unit (ICU) for the management of AP.
\end{abstract}

Methods: Using a multicentre, retrospective (2009-2014), observational database of ICU patients admitted for AP, our primary objective was to assess the incidence of AIT prescribing practices during the first 30 days following admission. Secondary objectives were to assess the independent impact of centre characteristics on the incidence of AIT and to identify factors associated with crude hospital mortality in a logistic regression model.

Results: In this cohort of 860 patients, 359 (42\%) received AIT on admission. Before day 30, 340/359 (95\%) AIT patients and 226/501 (45\%) AlT-free patients on admission received additional AIT, mainly for intra-abdominal and lung infections. A large heterogeneity was observed between centres in terms of the incidence of infections, therapeutic management including AIT and prognosis. Administration of AIT on admission or until day 30 was not associated with an increased mortality rate. Patients receiving AIT on admission had increased rates of complications (septic shock, intra-abdominal and pulmonary infections), therapeutic (surgical, percutaneous, endoscopic) interventions and increased length of ICU stay compared to AIT-free patients. Patients receiving delayed AIT after admission and until day 30 had increased rates of complications (respiratory distress syndrome, intra-abdominal and pulmonary infections), therapeutic interventions and increased length of ICU stay compared to those receiving AIT on admission. Risk factors for hospital mortality assessed on admission were age (adjusted odds ratio [95\% confidence interval] 1.03 [1.021.05]; $p<0.0001)$, Balthazar score $E(2.26$ [1.43-3.56]; $p<0.0001)$, oliguria/anuria (2.18 [1.82-4.33]; $p<0.0001)$, vasoactive support $(2.83[1.73-4.62] ; p<0.0001)$ and mechanical ventilation (1.90 [1.15-3.14]; $p=0.011)$, but not AIT (0.63 [0.40-1.01]; $p=0.057)$.

Conclusions: High proportions of ICU patients admitted for AP receive AIT, both on admission and during their ICU stay. A large heterogeneity was observed between centres in terms of incidence of infections, AIT prescribing practices, therapeutic management and outcome. AIT reflects the initial severity and complications of $A P$, but is not a risk factor for death.

Keywords: Acute pancreatitis, Intensive care unit, Anti-infective therapy, Carbapenems, Mortality

\footnotetext{
* Correspondence: philippe.montravers@aphp.fr

'Département d'Anesthésie-Réanimation, CHU Bichat-Claude Bernard,

HUPNVS, APHP, 48 rue Henri Huchard, F-75018 Paris, France

${ }^{2}$ Université de Paris, Paris, France

Full list of author information is available at the end of the article
}

(c) The Author(s). 2019 Open Access This article is distributed under the terms of the Creative Commons Attribution 4.0 International License (http://creativecommons.org/licenses/by/4.0/), which permits unrestricted use, distribution, and reproduction in any medium, provided you give appropriate credit to the original author(s) and the source, provide a link to the Creative Commons license, and indicate if changes were made. The Creative Commons Public Domain Dedication waiver (http://creativecommons.org/publicdomain/zero/1.0/) applies to the data made available in this article, unless otherwise stated. 


\section{Introduction}

Limited data are available concerning empirical and documented anti-infective therapy (AIT) prescribing practices in patients admitted to the intensive care unit (ICU) for management of acute pancreatitis (AP). Many clinical conditions related to abdominal or extraabdominal sources of infection can lead to the prescription of AIT. Recent international guidelines recommend limiting the use of antibiotics $(\mathrm{AB})$ to cases of suspected necrotizing pancreatitis or nosocomial extra-pancreatic infection and to treat other known fungal infections with antifungal therapy (AF) $[1,2]$.

Most publications focusing on AIT in patients with AP have reported single-centre experiences [3-8], while multicentre data on the clinical and microbiological features of acute infections in ICU patients are rare. The largest multicentre point-prevalence study collected data from ICU patients one decade ago during the EPIC II trial [9]. The authors reported that half of these patients were infected and $71 \%$ received antibiotics on the day of the study.

We used data from a large multicentre retrospective database of ICU patients with AP [10] to describe AIT use on ICU admission and during the first 30 days, evaluate between-centre variability in terms of the incidence of infections and AIT prescribing practices, evaluate outcome in terms of morbidity and mortality and identify independent risk factors evaluated on admission associated with mortality.

\section{Methods}

Patient data were extracted from a multicentre, retrospective, observational database involving 17 French and Belgian ICUs [10]. Patients for whom data concerning AIT with curative intent were available from ICU admission until day 30 were selected. AIT administered before ICU admission could not be determined.

This study was approved by the French Society of Anaesthesiology and Critical Care Medicine Ethics Committee (00010254-2015-017) and the French Personal Data Protection Agency (16-023). According to French legislation, this observational study did not require the patients' informed consent. The study was performed in accordance with the STROBE recommendations [10].

\section{Clinical data}

Baseline demographic, clinical and laboratory characteristics; organ failure; AIT; and organ support therapies were recorded on ICU admission and until day 30. Diagnostic criteria for sepsis and septic shock were those used at the time of admission of the patients in agreement with the International Sepsis Definitions Conference [11]. Organ failures, scored according to the SOFA score, were used to describe severity on ICU admission
[12]. Commonly reported risk factors for AP [1] and Balthazar score were assessed on admission, while the BISAP score was calculated retrospectively [13]. Clinical management, microbiological examinations, criteria for diagnosis of infection and selection of AIT were decided according to local protocols based on the recommendations of the French Society of Anaesthesiology and Critical Care Medicine [14].

We analysed patients receiving AIT on ICU admission (day 0 ) and during the first 30 days (day $>0$ to day 30) of management, and AIT duration was recorded. Patients who did not receive any AIT at any time during the 30day follow-up period were classified as AIT-free. AIT with curative intent on day 0 was defined as empirical or documented AIT [15], and the type of antibiotic (betalactams, carbapenems, aminoglycosides, and anti-Grampositive agents) and antifungal (echinocandins and azoles) therapies were analysed on day 0 and during the first 30 days.

\section{Outcomes}

The primary objective of our study was to assess the incidence of AIT during the first 30 days following admission for AP. Secondary objectives were to assess the following: (i) the independent impact of centre characteristics on the incidence of AIT, (ii) all-cause mortality at hospital discharge and (iii) factors associated with crude hospital mortality.

In line with these objectives, the primary study endpoints were the proportions of patients receiving AIT during the first 30 days following admission, with a focus on pancreatic and extra-pancreatic infections and AIT use across specific conditions. Secondary endpoints were infectious complications of AP (e.g. organ failure, sepsis, surgical complications), therapeutic interventions (surgical, percutaneous, endoscopic) from baseline to day 30, all-cause mortality at hospital discharge and assessment of risk factors of death.

\section{Statistical analysis}

Results are expressed as median and interquartile range $[\mathrm{IQR}]$ or number and proportions. The chi-square test and Fisher's exact test were used to compare discrete variables, and unpaired Wilcoxon tests were used to compare quantitative variables.

The purpose of this study was strictly exploratory. We therefore chose not to take inflation of the alpha risk into account. For the same purpose, only one multivariate model was constructed for the overall population to investigate the association between mortality and the variables of interest. Risk factors for death were assessed by univariate analysis, and unadjusted odds ratio (OR) and $95 \%$ confidence intervals $(\mathrm{CI})$ were calculated. Variables with $p<0.10$ on univariate analysis were 
introduced as predictive factors into the complete-case multivariate logistic regression analyses using a backward selection method. The centre effect and AIT on ICU admission were forced into these analyses. The collinearity between predictors was analysed, but no sensitivity analyses were performed. The BISAP score was not used in these analyses because of its post hoc calculation. A logistic model was evaluated for discrimination with the C-statistic and for calibration with the HosmerLemeshow test. No special treatment was performed for missing variables. Statistical analysis was performed with SAS@ 9.4 (SAS Institute, Cary, NC, USA).

\section{Results}

\section{Study population}

Over a 50-month period (2009-2014), individual data for 1003 patients with a diagnosis of AP were collected from medical records on ICU admission and for the first 30 days of ICU stay or until early discharge or death. From this cohort, 860 patients for whom information on AIT was available were analysed (Fig. 1). Overall, 275 (32\%) patients did not receive any AIT during their ICU stay. These patients had a short ICU stay and predominantly presented less severe disease than patients receiving AIT (Table 1).

\section{Anti-infective therapy on ICU admission}

At the time of ICU admission, 359/860 (42\%) patients were receiving AIT with curative intent, while no cases of prophylactic AIT were reported (Fig. 1). No difference was observed between patients receiving AIT on day 0 and AIT-free patients on admission in terms of either demographic data or risk factors for AP, while patients receiving AIT presented criteria of more severe disease and more intensive baseline therapy (Table 2).

Most patients $(n=299 / 359(83 \%))$ with AIT on day 0 received empirical $A B$ therapy, while 60 (17\%) cases received documented $\mathrm{AB}$ therapy (Additional file 3: Table S1), with marked between-centre variability (range 9$64 \%)$. In three centres, more than $25 \%$ of cases were receiving documented AIT on day 0. Patients receiving documented $A B$ had less severe disease than those receiving empirical AIT, but they received similar proportions of broad-spectrum $A B$ and higher proportions of AF (Additional file 3: Table S1).

On day 0 , the indications for AIT varied among centres (Additional file 1: Figure S1). The predominant indications for AIT were intra-abdominal infections $(n=207$ patients, including $173(58 \%)$ receiving empirical therapy), pulmonary infections $(n=46$, empirical therapy $n=41(14 \%))$ and bacteraemia $(n=43$,

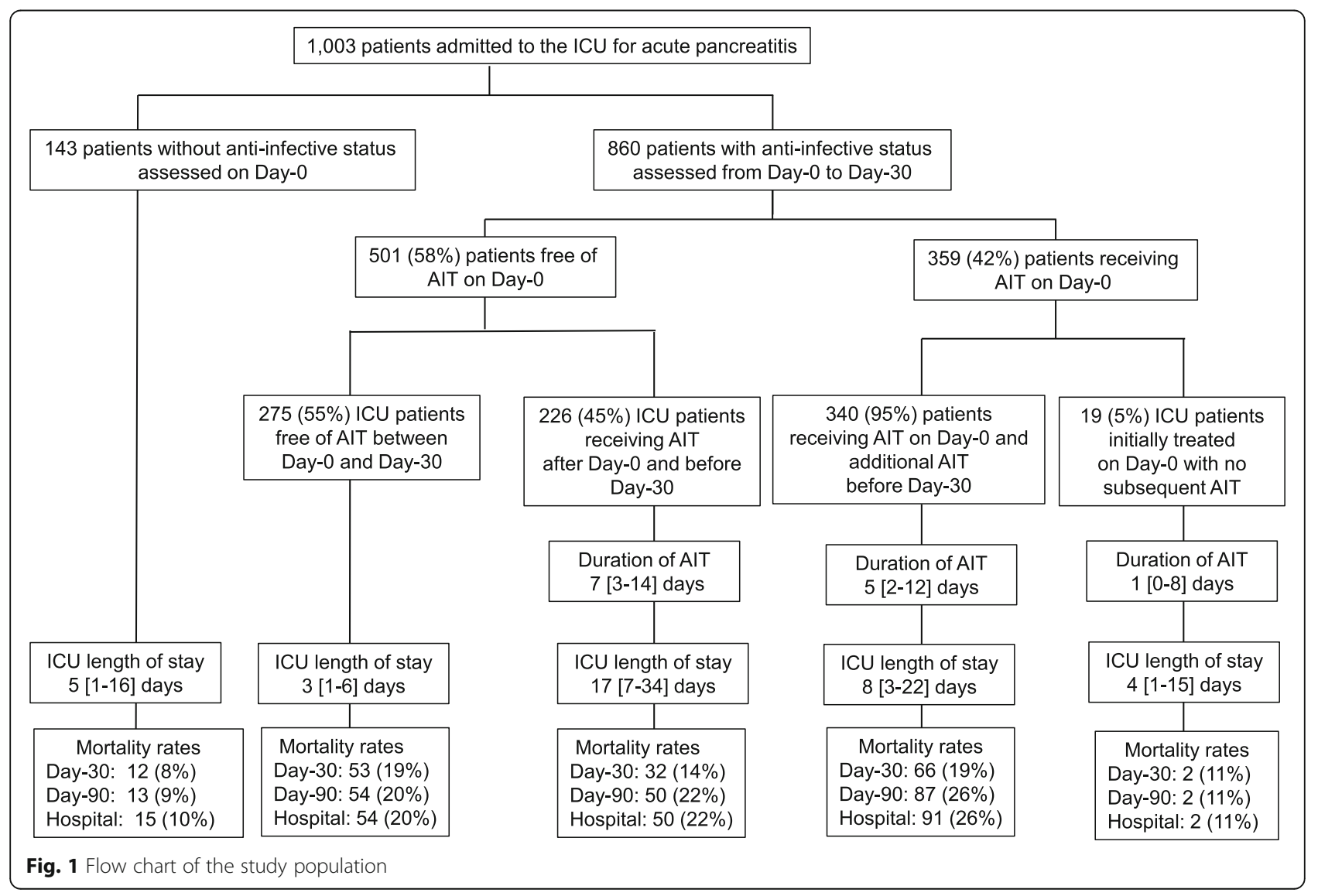


Table 1 Clinical features and prognosis for the AIT-free patients and those receiving AIT

\begin{tabular}{|c|c|c|c|c|}
\hline & Missing data & AlT-free patients $n=275$ & $\begin{array}{l}\text { Patients receiving AIT } \\
\text { during their ICU stay } n=585\end{array}$ & $p$ value \\
\hline Male, $n(\%)$ & $1 / 4$ & $166(61)$ & $386(66)$ & NS \\
\hline Age, years, median [IQR] & $3 / 4$ & $55[40-71]$ & $59[48-72]$ & $<0.01$ \\
\hline \multicolumn{5}{|l|}{ Underlying diseases } \\
\hline Diabetes, $n(\%)$ & 0 & $45(16)$ & $91(16)$ & NS \\
\hline Cardiovascular disease, $n$ (\%) & 0 & $157(57)$ & $342(58)$ & NS \\
\hline Respiratory disease, $n$ (\%) & 0 & $44(16)$ & $100(17)$ & NS \\
\hline Renal disease, $n(\%)$ & 0 & $24(9)$ & $31(5)$ & NS \\
\hline Liver disease, $n(\%)$ & $0 / 1$ & $30(11)$ & $63(11)$ & NS \\
\hline Smoking, $n(\%)$ & 0 & $137(50)$ & $251(43)$ & NS \\
\hline Alcoholism, n (\%) & $0 / 1$ & $128(47)$ & $247(42)$ & NS \\
\hline \multicolumn{5}{|l|}{ Attributable cause of pancreatitis } \\
\hline Alcoholism, n (\%) & 0 & $80(29)$ & $146(25)$ & NS \\
\hline Gallstones, $n(\%)$ & 0 & $97(35)$ & $247(42)$ & NS \\
\hline Post-ERCP, $n(\%)$ & 0 & $9(3)$ & $47(8)$ & $<0.01$ \\
\hline Hypertriglyceridaemia, n (\%) & 0 & $26(9)$ & $31(5)$ & $<0.05$ \\
\hline Cancer, $n(\%)$ & 0 & $8(3)$ & $6(1)$ & NS \\
\hline Toxic, $n(\%)$ & 0 & $4(1)$ & $17(3)$ & NS \\
\hline Undetermined, $n(\%)$ & 0 & $51(19)$ & $96(16)$ & NS \\
\hline \multicolumn{5}{|l|}{ Clinical presentation on admission } \\
\hline Pain onset/ICU admission interval, days, median [IQR] & 0 & $2[1-3]$ & $3[1-8]$ & $<0.001$ \\
\hline Oliguria/anuria, $n(\%)$ & 0 & $120(44)$ & $253(43)$ & NS \\
\hline BISAP score, median [IQR] & $12 / 22$ & $1[0-1]$ & $1[1-2]$ & $<0.001$ \\
\hline Balthazar score $\mathrm{E}, n(\%)$ & 0 & $137(50)$ & $356(61)$ & $<0.01$ \\
\hline \multicolumn{5}{|l|}{ Severity criteria on admission } \\
\hline SOFA score, median [IQR] & $9 / 14$ & $3[1-5]$ & $5[3-7]$ & $<0.001$ \\
\hline Respiratory failure*, $n$ (\%) & 0 & $39(14)$ & $207(35)$ & $<0.001$ \\
\hline Cardiovascular failure*,$n(\%)$ & 0 & $37(13)$ & $202(35)$ & $<0.001$ \\
\hline Renal failure* $n$ (\%) & 0 & $43(16)$ & $100(17)$ & NS \\
\hline Septic shock, $n(\%)$ & 0 & $3(1)$ & $114(19)$ & $<0.001$ \\
\hline Acute respiratory distress syndrome, $n(\%)$ & 0 & $9(3)$ & $53(9)$ & $<0.01$ \\
\hline \multicolumn{5}{|l|}{ Therapeutic management on admission } \\
\hline Vasoactive support, $n$ (\%) & $0 / 1$ & $38(14)$ & $207(35)$ & $<0.001$ \\
\hline Mechanical ventilation, $n$ (\%) & $1 / 5$ & $38(14)$ & $187(32)$ & $<0.001$ \\
\hline Fluid loading, $n(\%)$ & $2 / 12$ & $178(65)$ & $447(78)$ & $<0.001$ \\
\hline Renal replacement therapy, n (\%) & $1 / 10$ & $16(6)$ & $62(11)$ & $<0.05$ \\
\hline \multicolumn{5}{|l|}{ Main treatments between day $>0$ and day 30} \\
\hline Need for red blood cell transfusion, $n(\%)$ & 0 & $31(11)$ & $195(33)$ & $<0.001$ \\
\hline Number of days of mechanical ventilation & $1 / 2$ & $0[0-0]$ & $4[0-16]$ & $<0.001$ \\
\hline Vasoactive support, $n$ (\%) & $0 / 1$ & $59(21)$ & $335(57)$ & $<0.001$ \\
\hline Renal replacement therapy, $n(\%)$ & $1 / 1$ & $42(15)$ & $178(30)$ & $<0.001$ \\
\hline Duration of RRT, days, median [IQR] & $14 / 0$ & $2[2-4]$ & $7[2-15]$ & $<0.001$ \\
\hline \multicolumn{5}{|l|}{ Main complications between day $>0$ and day 30} \\
\hline Acute respiratory distress syndrome, $n(\%)$ & $0 / 2$ & $40(15)$ & $201(34)$ & $<0.001$ \\
\hline
\end{tabular}


Table 1 Clinical features and prognosis for the AIT-free patients and those receiving AIT (Continued)

\begin{tabular}{|c|c|c|c|c|}
\hline & Missing data & AlT-free patients $n=275$ & $\begin{array}{l}\text { Patients receiving AIT } \\
\text { during their ICU stay } n=585\end{array}$ & $p$ value \\
\hline Septic shock, $n(\%)$ & $24 / 47$ & $4(2)$ & $210(39)$ & $<0.001$ \\
\hline Pancreatic necrosis, $n$ (\%) & $12 / 18$ & $164(62)$ & $393(69)$ & $<0.05$ \\
\hline Infected necrosis, $n$ (\%) & $15 / 29$ & $5(2)$ & $200(36)$ & $<0.001$ \\
\hline Gastro-intestinal perforation, $n(\%)$ & $12 / 32$ & $4(1)$ & $50(9)$ & $<0.001$ \\
\hline Vascular thrombosis, $n(\%)$ & $12 / 26$ & $22(8)$ & $86(15)$ & $<0.01$ \\
\hline Acute mesenteric ischaemia, $n(\%)$ & $12 / 28$ & $25(10)$ & $63(11)$ & NS \\
\hline Intra-abdominal collection, $n(\%)$ & $10 / 22$ & $37(14)$ & $242(43)$ & $<0.001$ \\
\hline Abdominal compartment syndrome, $n(\%)$ & $12 / 26$ & $17(6)$ & $66(12)$ & $<0.05$ \\
\hline Haemorrhage, $n$ (\%) & $11 / 26$ & $19(7)$ & $81(14)$ & $<0.01$ \\
\hline Peritonitis, $n(\%)$ & $12 / 30$ & $3(1)$ & $85(15)$ & $<0.001$ \\
\hline Cholangitis, $n(\%)$ & $15 / 25$ & $2(1)$ & $57(10)$ & $<0.001$ \\
\hline Digestive fistula, $n(\%)$ & $12 / 28$ & $1(1)$ & $30(5)$ & $<0.001$ \\
\hline \multicolumn{5}{|c|}{ Clinical management between day $>0$ and day 30} \\
\hline Endoscopic necrosectomy, $n$ (\%) & $2 / 6$ & $24(9)$ & $152(26)$ & $<0.001$ \\
\hline Surgical necrosectomy, $n$ (\%) & $0 / 6$ & $32(12)$ & $219(38)$ & $<0.001$ \\
\hline Radiological drainage, $n(\%)$ & $0 / 6$ & $8(3)$ & $120(21)$ & $<0.001$ \\
\hline Duration of ICU stay, days, median [IQR] & 0 & $3[1-6]$ & $12[4-27]$ & $<0.001$ \\
\hline ICU readmission, $n(\%)$ & $0 / 1$ & $6(2)$ & $50(9)$ & $<0.001$ \\
\hline Hospital mortality rate, $n(\%)$ & 0 & $54(20)$ & $143(24)$ & NS \\
\hline Time to death, days, median [IQR] & 0 & $1.5[1-2]$ & $12[2-34]$ & $<0.001$ \\
\hline
\end{tabular}

NS non-significant, NA not applicable, ERCP endoscopic retrograde cholangiopancreatography

*According to the definition of the SOFA score

empirical therapy $n=28$ (9\%)). Among the other sources of infections, low proportions of catheterrelated infections, urinary tract infections and skin and soft tissue infections were also recorded (Fig. 2a). Overall, mixed sources of infection in patients with intra-abdominal infections were reported in $22(11 \%)$ cases, including 11 bacteraemic intra-abdominal infections and 8 cases with combined intra-abdominal and pulmonary infections (Fig. 2a). Among the 46 (13\%) cases initially treated for pneumonia, 3 cases of bacteraemia were observed.

A large inter-centre variability was noted in terms of frequency and type of AIT agents (Additional file 1: Figure S2 and S3). Beta-lactams were the agents predominantly used in 272 (76\%) patients, including carbapenems $(n=81(23 \%))$. The other most frequently prescribed agents were aminoglycosides $(n=120(33 \%))$ and antiGram-positive agents $(n=51(14 \%))$ (Fig. 2a). The 81 patients receiving carbapenems were not different from the 278 patients receiving other AIT in terms of severity, but more frequently received combination therapy comprising aminoglycosides, anti-Gram-positive and antifungal therapies (Table 3). Interestingly, only $14 / 81$ (17\%) of these carbapenem prescriptions were documented indications.
Baseline AF therapy was reported in 53 patients (Fig. 2a, Additional file 1: Figure S2) with a predominance of azoles $(n=46(13 \%$ of all AIT) $)$ and 5 prescriptions of echinocandins. These patients did not differ from those receiving $A B$ therapy in terms of underlying disease, initial severity or organ failure. Initial AF therapy was always administered in combination with $A B$ therapy, mainly empirical $(n=36)$ and frequently comprising carbapenems (20/53 (38\%) versus 61/306 (20\%) for those receiving $\mathrm{AB}$ therapy, $p<0.05$ ). The predominant indications for AF were intra-abdominal infections $(n=37(70 \%))$ and bacteraemia $(n=12(23 \%))$.

\section{Anti-infective therapy between day $>0$ and day 30}

Among the 359 patients receiving AIT on day 0, 19 patients did not receive any subsequent course of AIT, while the remaining 340 cases required additional AIT regimens (Figs. 1 and 2b). Overall, between day $>0$ and day 30 , AIT was administered to $566 / 860$ (66\%) patients, including 226 patients who were AIT-free on day 0 (Figs. 1 and 2c). No difference was observed between these two subgroups of patients in terms of underlying diseases and cause of AP. Compared to the 340 patients who received AIT from day 0, the 226 cases with delayed AIT had a less severe clinical presentation on admission. 
Table 2 Clinical features for the patients with/without anti-infective therapy on ICU admission (day 0)

\begin{tabular}{|c|c|c|c|c|}
\hline & Missing data & AlT-free patients on day $0 n=501$ & Patients receiving AIT on day $0 n=359$ & $p$ value \\
\hline Male, $n(\%)$ & $0 / 5$ & $313(62)$ & $239(68)$ & NS \\
\hline Age, years, median [IQR] & $3 / 4$ & $56[43-71]$ & 60 [49-73] & $<0.01$ \\
\hline \multicolumn{5}{|l|}{ Clinical presentation on admission } \\
\hline $\begin{array}{l}\text { Pain onset/ICU admission interval, days, } \\
\text { median [IQR] }\end{array}$ & 0 & $2[1-4]$ & $4[1-11]$ & $<0.001$ \\
\hline Oliguria/anuria, $n$ (\%) & 0 & $219(44)$ & $154(43)$ & NS \\
\hline BISAP score, median [IQR] & $16 / 18$ & $1[0-1]$ & $2[1-2]$ & $<0.001$ \\
\hline Balthazar score $\mathrm{E}, n(\%)$ & 0 & $293(58)$ & $200(56)$ & NS \\
\hline \multicolumn{5}{|l|}{ Severity criteria on admission } \\
\hline SOFA score, median [IQR] & $10 / 12$ & $4[2-6]$ & $5[3-7]$ & $<0.001$ \\
\hline Respiratory failure*, $n$ (\%) & 0 & $109(22)$ & $137(38)$ & $<0.001$ \\
\hline Cardiovascular failure*,$n(\%)$ & 0 & $77(15)$ & $162(45)$ & $<0.001$ \\
\hline Renal failure*,$n(\%)$ & 0 & $90(18)$ & $53(15)$ & NS \\
\hline Septic shock, $n(\%)$ & 0 & $7(1)$ & $110(31)$ & $<0.001$ \\
\hline Acute respiratory distress syndrome, $n(\%)$ & 0 & $25(5)$ & $37(10)$ & $<0.01$ \\
\hline \multicolumn{5}{|l|}{ Therapeutic management on admission } \\
\hline Vasoactive support, $n(\%)$ & $0 / 1$ & $80(16)$ & $165(46)$ & $<0.001$ \\
\hline Mechanical ventilation, $n(\%)$ & $1 / 5$ & $95(19)$ & $130(37)$ & $<0.001$ \\
\hline Fluid loading, $n(\%)$ & $2 / 12$ & $348(70)$ & $277(80)$ & $<0.01$ \\
\hline Renal replacement therapy, $n(\%)$ & $1 / 10$ & $36(7)$ & $42(12)$ & $<0.05$ \\
\hline Anti-infective therapy, $n$ (\%) & 0 & - & $359(100)$ & $<0.001$ \\
\hline \multicolumn{5}{|l|}{ Main treatments between day $>0$ and day 30} \\
\hline Need for red blood cell transfusion, $n(\%)$ & 0 & $110(22)$ & $116(32)$ & $<0.001$ \\
\hline Number of days of mechanical ventilation & $2 / 8$ & $0[0-9]$ & $2[0-10]$ & $<0.001$ \\
\hline Vasoactive support, $n(\%)$ & $0 / 1$ & $186(37)$ & $208(58)$ & $<0.001$ \\
\hline Renal replacement therapy, $n(\%)$ & $2 / 0$ & $127(25)$ & $93(26)$ & NS \\
\hline Duration of RRT, days, median [IQR] & $6 / 9$ & $5[2-14]$ & $5[2-15]$ & NS \\
\hline \multicolumn{5}{|l|}{ Main complications between day $>0$ and day 30} \\
\hline Acute respiratory distress syndrome, $n(\%)$ & $0 / 2$ & $142(28)$ & $99(28)$ & NS \\
\hline Septic shock, $n(\%)$ & $39 / 32$ & $90(19)$ & $124(38)$ & 0.001 \\
\hline Pancreatic necrosis, $n$ (\%) & $13 / 17$ & $339(69)$ & $218(64)$ & NS \\
\hline Infected necrosis, $n$ (\%) & $20 / 24$ & $88(18)$ & $117(35)$ & $<0.001$ \\
\hline Gastro-intestinal perforation, $n(\%)$ & $20 / 24$ & $27(6)$ & $27(8)$ & NS \\
\hline Vascular thrombosis, $n$ (\%) & $16 / 22$ & $55(11)$ & $53(16)$ & NS \\
\hline Acute mesenteric ischaemia, $n(\%)$ & $18 / 22$ & $52(11)$ & $36(11)$ & NS \\
\hline Intra-abdominal collection, $n(\%)$ & $14 / 18$ & $127(26)$ & $152(45)$ & $<0.001$ \\
\hline Abdominal compartment syndrome, $n(\%)$ & $16 / 22$ & $52(11)$ & $31(9)$ & NS \\
\hline Haemorrhage, $n(\%)$ & $16 / 21$ & $53(11)$ & $47(14)$ & NS \\
\hline Peritonitis, $n(\%)$ & $19 / 23$ & $29(6)$ & $59(18)$ & $<0.001$ \\
\hline Cholangitis, n (\%) & $21 / 19$ & $18(4)$ & $41(12)$ & $<0.001$ \\
\hline Digestive fistula, $n(\%)$ & $19 / 21$ & $11(2)$ & $20(6)$ & $<0.01$ \\
\hline \multicolumn{5}{|l|}{ Clinical management between day $>0$ and day 30} \\
\hline Endoscopic necrosectomy, $n(\%)$ & $3 / 5$ & $89(18)$ & $87(25)$ & $<0.05$ \\
\hline Surgical necrosectomy, n (\%) & $1 / 5$ & $119(24)$ & $132(37)$ & $<0.001$ \\
\hline
\end{tabular}


Table 2 Clinical features for the patients with/without anti-infective therapy on ICU admission (day 0) (Continued)

\begin{tabular}{|c|c|c|c|c|}
\hline & Missing data & AlT-free patients on day $0 n=501$ & Patients receiving AIT on day $0 n=359$ & $p$ value \\
\hline Radiological drainage, $n(\%)$ & $1 / 5$ & $56(11)$ & $72(20)$ & $<0.001$ \\
\hline Duration of ICU stay, days, median [IQR] & 0 & $6[2-17]$ & $8[3-21]$ & $<0.05$ \\
\hline ICU readmission $n(\%)$ & $0 / 1$ & $30(6)$ & $26(7)$ & NS \\
\hline Hospital mortality rate, $n(\%)$ & 0 & $104(21)$ & $93(26)$ & NS \\
\hline Time to death, days, median [IQR] & 0 & $3[1-18]$ & $7[1-31]$ & NS \\
\hline
\end{tabular}

NS non-significant, NA not applicable, AIT anti-infective therapy, ERCP endoscopic retrograde cholangiopancreatography ${ }^{*}$ According to the definition of the SOFA score

In addition, these patients subsequently experienced a more complicated course with prolonged ICU stay and prolonged AIT (Table 4 and Fig. 1).

Most patients received $A B$, with a marked variability between centres (median 76\% (range 54-91) of patients). The indications for AIT varied among centres, but the predominant indications remained intra-abdominal and pulmonary infections (Fig. 2b, c and Additional file 1: Figure S1, S2, and S3). Mixed sources of infections were reported in $98 / 340$ (29\%) patients treated on day 0 and receiving additional AIT before day 30 and 84/226 (37\%) patients receiving delayed therapy after ICU admission (Fig. 2b, c).

Carbapenems and anti-Gram-positive agents were frequently prescribed between day $>0$ and day 30, representing 202/566 (36\%) and 161/566 (28\%) of all AIT prescriptions, respectively (Fig. 2b, c). Patients receiving carbapenems between day 0 and day 30 had more severe disease than those treated by other AITs. These patients received more combination AIT and had a higher level of therapeutic management than those receiving other AIT, including endoscopic/radiological and surgical drainage (Table 5).

Antifungal therapy was reported in 110/566 (19\%) patients with a predominance of azoles $(n=67(61 \%))$ for intra-abdominal indications $(n=76(70 \%))$ with a large between-centre variability (median of $11 \%$ of cases (range 0-31\%) (Additional file 1: Figure S2).

A marked between-centre variability of therapeutic interventions was also reported between day $>0$ and day 30 , illustrated by large variations in the proportions of patients who underwent endoscopic necrosectomy, surgical necrosectomy and percutaneous drainages (Additional file 2: Figure S4).

\section{Outcome}

Patients receiving AIT from ICU admission had more infectious complications and therapeutic interventions (both medical and surgical) during the first 30 days of care than AIT-free patients on day 0 (Table 2). The 226 cases with delayed AIT had more complications, increased rates of medical management (prolonged duration of mechanical ventilation and AIT, increased frequency of renal replacement therapy) and increased duration of ICU stay, but similar proportions of surgical management compared to the 340 patients who received AIT from day 0 (Table 4). In addition, the patients receiving carbapenems between day $>0$ and day 30 had more infectious complications and more therapeutic interventions (both medical and surgical) than the 364 cases receiving other AITs (Table 5).

Overall, 197/860 (23\%) patients died after a median interval of 4 [1-27] days. The hospital mortality rate was not significantly different between patients receiving AIT and AIT-free patients between day 0 and day 30 (Table 1). Fifty-four (20\%) of these AIT-free patients died after a median [IQR] of $1.5[1,2]$ days. Most of these patients had underlying cardiovascular diseases $(n=42(78 \%))$ and were admitted for severe AP (Balthazar score E $n=42(78 \%))$. From ICU admission, they received mechanical ventilation $(n=27$ (50\%)), vasoactive support $(n=26(48 \%))$ and renal replacement therapy $(n=12(22 \%))$. Major events in the clinical course of these patients included pancreatic necrosis $(n=45(87 \%))$, mesenteric ischaemia $(n=22$ (42\%)), abdominal compartment syndrome $(n=9$ $(17 \%))$ and/or vascular thrombosis $(n=8(15 \%))$.

Mortality rates were not different between patients treated on ICU admission and those receiving delayed AIT (Table 4). Interestingly, the outcome of patients receiving carbapenems on day 0 was not different from that of those receiving other AITs (Table 3), while patients receiving carbapenems between day $>0$ and day 30 had a poorer outcome with a higher mortality rate (Table 5). Among the 205 patients with infected pancreatic necrosis, 56 (27\%) died after a median [IQR] of 31 [10-44] days compared to 4 [1$19]$ days for the 141 patients who died without pancreatic necrosis $(p<0.001)$. A marked between-centre variability of mortality rates (range $0-41 \%$ ) was reported, illustrated by 3 centres with mortality rates higher than $35 \%$ and 3 centres with mortality rates lower than 10\% (Additional file 1: Figure S5). Comparison of the clinical presentation, management and outcome of the patients admitted to these units is presented in Additional file 3: Table S2. 


\begin{tabular}{|c|c|}
\hline 359 patients receiving AIT on Day- 0 & Type of anti-infective therapy administered on Day- 0 \\
\hline \multicolumn{2}{|l|}{ Intra-abdominal site of infection ( $n=207$ patients) } \\
\hline Single intra-abdominal infection $(n=185)$ & $\begin{array}{l}\text { (BL } n=158(C P n=47)+A G n=66 \text { or } A G P n=27 \text { or } A F n=17) \\
\text { (Other regimens } n=27 \text { including } A G n=9, A G P n=4, A F n=7)\end{array}$ \\
\hline \multirow{2}{*}{$\begin{array}{l}\text { Pulmonary site of infection ( } n=38 \text { patients) } \\
\text { Single pulmonary infection }(n=34)\end{array}$} & $\begin{array}{l}\text { Pulmonary infection } n=8(B L n=6(C P n=3)+A G n=4 \text { or } A G P n=1 \text { or } \\
\text { AF } n=3) \\
\text { Bacteraemia } n=7 \text { (BL } n=5(C P n=3), A G n=1, A G P=1 \text { or } A F n=1) \\
\text { CRI } n=1+\text { bacteraemia } n=3 \text { (Other regimens including AF } n=3) \\
\text { UTI } n=1 \text { + bacteraemia } n=1 \text { ((Other regimens including AF } n=2) \\
\text { SSTI } n=1 \text { (Other regimen including AF } n=1)\end{array}$ \\
\hline & $\begin{array}{l}(B L n=29(C P n=4)+A G n=8 \text { or } A G P n=1) \\
\text { (Other regimens } n=5 \text { including } A G P n=1)\end{array}$ \\
\hline other sites $(n=4)$ & $\begin{array}{l}\text { Bacteraemia } n=3 \text { (Other regimens including } A G n=1) \\
\text { UTI } n=1(B L n=1)\end{array}$ \\
\hline \multirow{2}{*}{$\begin{array}{l}\text { Urinary site of infection }(n=6 \text { patients) } \\
\text { Single urinary tract infection }(n=4)\end{array}$} & $\begin{array}{l}\text { (BL } n=17 \text { (CP } n=2 \text { ) plus } A G n=5 \text { or } A G P n=3 \text { or } A F n=1 \text { ) } \\
\text { (Other regimens including } A G n=2, A G P n=5, A F n=3 \text { ) }\end{array}$ \\
\hline & (Other regimens including $B L n=1, A G n=1$ ) \\
\hline \multirow[b]{2}{*}{$\begin{array}{l}\text { Catheter-related site of infection ( } n=4 \text { patients) } \\
\text { Single catheter-related infection }(n=2)\end{array}$} & Bacteraemia $n=2(B L n=1, A G n=1, A F n=1)$ \\
\hline & $(B L n=2(C P n=1)+A G n=1)$ \\
\hline Associated to other sites $(n=2)-$ & Bacteraemia $n=2(B L n=2(C P n=1)+A G n=1+A G P n=1)$ \\
\hline $\begin{array}{r}\text { Skin and soft tissue site of infection (n=1 patient) } \\
\text { Associated to other sites (n=1) }\end{array}$ & Bacteraemia $n=1$ (Other regimen including AF $n=1$ ) \\
\hline $\begin{array}{l}\text { Missing information on the source of infection- } \\
\qquad(\mathrm{n}=76 \text { patients) }\end{array}$ & $\begin{array}{l}\text { (Other regimens including BL } n=48 \text { (CP } n=18), A G n=17, A G P n=7, A F \\
n=12)\end{array}$ \\
\hline
\end{tabular}

\section{A}

\begin{tabular}{|c|c|}
\hline $\begin{array}{l}340 \text { patients treated on Day- } 0 \\
\text { and receiving additional AIT before Day- } 30\end{array}$ & $\begin{array}{l}\text { Type of anti-infective therapy } \\
\text { administered before Day-30 }\end{array}$ \\
\hline \multicolumn{2}{|l|}{$\begin{array}{l}\text { Intra-abdominal site of infection (n=219 patients) } \\
\text { Single intra-abdominal infection }(n=137)-\end{array}$} \\
\hline & $\begin{array}{l}\text { (CP } n=46+A G n=24 \text { or } A G P n=13 \text { or } A F n=9 \text { ) } \\
\text { (Other regimens } n=91 \text { including } A G n=42 \text { or } A G P=17 \text { or } A F n=11 \text { ) }\end{array}$ \\
\hline Associated to other sites $(n=82)$ & $\begin{array}{l}\text { Pulmonary infection } n=44 \text { (CP } n=31, A G n=26, A G P n=24 \text { or AF } n=17) \\
\text { Bacteraemia } n=26(C P n=14, A G n=11, A G P n=17, A F n=9) \\
\text { CRI } n=1 \text { (Other regimen } n=1) \\
\text { UTI } n=1(A F n=1) \\
\text { SSTI } n=2(+ \text { bacteraemia } n=1 \text {, Pulmonary infection } n=1) \text { (CP } n=1, A G \\
n=1, A G P n=1, A F n=1)\end{array}$ \\
\hline Single pulmonary infection $(n=$ & (Other regimens including $C P n=5, A G n=6, A G P n=4$ ) \\
\hline Associated to other sites ( $n=12)$ & $\begin{array}{l}\text { Bacteraemia } n=7 \text { (CP } n=3, A G n=3, A G P n=3, A F n=2) \\
\text { UTI } n=4 \text { (Other regimens including CP } n=1, A G n=1, A G P n=1 \text { ) } \\
\text { SSTI } n=1 \text { (+UTI) (Other regimen } n=1 \text { ) }\end{array}$ \\
\hline Primary bacteraemia $(n=26 p$ & $(\mathrm{CP} n=4, \mathrm{AG} n=3, \mathrm{AGP} n=6)$ \\
\hline Urinary site of infecti & \\
\hline 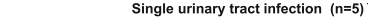 & (Other regimens including $C P n=1, A G n=1, A F n=2$ ) \\
\hline Associated to other sites ( $\mathrm{n}$ & Bacteraemia and CRI $n=1(A G P n=1)$ \\
\hline $\begin{array}{l}\text { Catheter-related site of infection ( } n=4 \text { patients) } \\
\text { Single catheter-related infection }(n=1)\end{array}$ & \\
\hline & Other regimen $\mathrm{n}=1$ \\
\hline Assoc & Bacteraemia $n=3$ (Other regimens including $C P n=1, A G n=1$ ) \\
\hline $\begin{array}{l}\text { Missing information on the source of } \\
\text { infection ( } n=55 \text { patients) }\end{array}$ & (Other regimens including $C P n=11, A G n=13, A G P n=7, A F n=11$ ) \\
\hline
\end{tabular}

B

\begin{tabular}{|c|c|}
\hline $\begin{array}{l}226 \text { patients free of AIT on Day-0 and } \\
\text { receiving AIT after Day- } 0 \text { and before Day- } 30\end{array}$ & $\begin{array}{l}\text { Type of anti-infective therapy } \\
\text { administered between Day>0 and Day-30 }\end{array}$ \\
\hline \multicolumn{2}{|l|}{$\begin{array}{l}\text { Intra-abdominal site of infection ( } n=130 \text { patients) } \\
\text { Single intra-abdominal infection }(n=63)\end{array}$} \\
\hline Pulmonary site of infection ( $n=39$ patients) & $\begin{array}{l}\text { Pulmonary infection } n=25(C P n=17, A G n=18, A G P n=12, A F n=8) \\
\text { Pulmonary infection } n=20(+ \text { bacteraemia } n=16+C R I n=8+U T I n=7) \\
(C P n=10, A G n=17, A G P n=13, A F n=7) \\
\text { Bacteraemia } n=10(C P n=5, A G n=5, A G P=2, A F n=2) \\
\text { Bactereaemia } n=7(+C R I n=6+U T I n=3)(C P n=5, A G n=2, A G P n=2, A F \\
n=3) \\
\text { CR } n=2 \text { (Other regimens including } C P n=1) \\
\text { UTI } n=3(C P n=1, A G n=1, A G P n=1)\end{array}$ \\
\hline & (Other regimens including $C P n=5, A G n=9, A G P n=4, A F n=3$ ) \\
\hline Associated to other sites $(n=11)$ & $\begin{array}{l}\text { Bacteraemia } n=5 \text { (CP } n=5, A G n=3, A G P n=3, A F n=1) \\
\text { UTI } n=2 \text { (Other regimens including AG } n=2 \text { ) } \\
\text { CRI } n=2 \text { (Other regimens) } \\
\text { SSII } n=2 \text { (+Bacteraemia +CRI + UTI n=1) (Other regimens including AG } \\
n=2 \text { ) }\end{array}$ \\
\hline $\begin{array}{l}\text { Primary bacteraemia ( } n=13 \text { patients }) \\
\text { Urinary site of infection ( } n=15 \text { patients) }\end{array}$ & (Other regimens including $C P n=2, A G n=2, A G P n=2, A F n=1$ ) \\
\hline & (Other regimens including $C P n=1, A G n=2, A G P n=1$ ) \\
\hline Associated to other sites $(n=4)$ & $\begin{array}{l}\text { Bacteraemia } n=2+\text { Catheter related infection } n=2 \\
\text { (Other regimens including } C P n=1, A G n=1, A G P n=1, A F n=1 \text { ) }\end{array}$ \\
\hline & (Other regimens $\mathrm{n}=3$ ) \\
\hline $\begin{array}{l}\text { Associated to other sites }(n=2) \\
\text { Skin and soft tissue source of infection }\end{array}$ & Bacteraemia $n=2(C P n=2, A G n=2, A G P n=1)$ \\
\hline $\begin{array}{c}(n=1 \text { patient) } \\
\text { Missing information on the source }\end{array}$ & (Other regimen $n=1$ ) \\
\hline $\begin{array}{l}g \text { information on the source } \\
\text { of infection ( } n=23 \text { patients) }\end{array}$ & (Other regimens including $C P n=9, A G n=11, A G P n=4, A F n=6$ ) \\
\hline
\end{tabular}

Fig. 2 (See legend on next page.) 
(See figure on previous page.)

Fig. 2 Most frequent sources of infection recorded and treated on admission in 359 patients (a), during the 30 days of follow-up for 340 of them (b) and in another 226 AlT-free patients on admission treated after day 0 and before day 30 (c). AF, antifungal therapy; AG, aminoglycosides; AGP, anti-Gram-positive agents; BL, beta-lactams; CP, carbapenems; CRI, catheter-related infection; UTI, urinary tract infection; SSTI, skin and soft tissue infection

In univariate analysis, a higher mortality rate was observed among patients with septic shock or pneumonia on day 0 , while AIT on day 0 did not influence the mortality rate (Fig. 3). In multivariate analysis, risk factors collected on admission and related to death were age, Balthazar score E, oliguria-anuria, vasoactive support and mechanical ventilation on admission, but not AIT (Fig. 4).

\section{Discussion}

To the best of our knowledge, this is currently the largest study assessing the use of AIT in ICU patients admitted for the management of AP. Overall, $42 \%$ of these patients received AIT on admission. Between day $>0$ and day $30,95 \%$ of these patients received an additional course of AIT, and $45 \%$ of patients who were AIT-free on admission subsequently received AIT. Our data suggest that patients who received early AIT at day 0 presented more severe disease than patients without AIT. In addition, patients receiving delayed AIT appeared to present a higher morbidity rate, despite the absence of significantly increased mortality. Major between-centre variability was observed in terms of both the incidence of infections and therapeutic management. Underlying diseases and baseline severity appeared to be the key

Table 3 Clinical features for the patients receiving carbapenems or other antibiotics on day 0

\begin{tabular}{|c|c|c|c|c|}
\hline & Missing data & Carbapenems $n=81$ & Other AIT $n=278$ & $p$ value \\
\hline \multicolumn{5}{|l|}{ Severity criteria at day 0} \\
\hline SOFA score, median [IQR] & $4 / 9$ & $5[3-7]$ & $5[3-7]$ & NS \\
\hline Respiratory failure*, $n$ (\%) & 0 & $32(40)$ & $103(37)$ & NS \\
\hline Cardiovascular failure*,$n(\%)$ & 0 & $39(48)$ & $123(44)$ & NS \\
\hline Renal failure*, $n(\%)$ & 0 & $14(17)$ & $53(19)$ & NS \\
\hline Septic shock, $n(\%)$ & 0 & $29(36)$ & $81(29)$ & NS \\
\hline Acute respiratory distress syndrome, $n(\%)$ & 0 & $11(14)$ & $26(9)$ & NS \\
\hline \multicolumn{5}{|l|}{ Main reasons for anti-infective therapy at day 0} \\
\hline Empirical therapy, $n(\%)$ & 0 & $67(83)$ & $232(83)$ & NS \\
\hline Intra-abdominal infection, $n$ (\%) & $1 / 5$ & $55(69)$ & $155(57)$ & NS \\
\hline Pneumonia, $n(\%)$ & $2 / 6$ & $7(9)$ & $39(14)$ & NS \\
\hline Bacteraemia, $n(\%)$ & $2 / 3$ & $6(8)$ & $39(14)$ & NS \\
\hline Catheter-related infection, $n(\%)$ & $2 / 3$ & $2(3)$ & $7(3)$ & NS \\
\hline Urinary tract infection, $n(\%)$ & $2 / 4$ & - & $9(3)$ & NS \\
\hline Skin and soft tissue infection, $n(\%)$ & $2 / 3$ & - & $2(1)$ & NS \\
\hline \multicolumn{5}{|c|}{ Most frequently prescribed anti-infective agents at day 0} \\
\hline Beta-lactams, $n(\%)$ & 0 & $81(100)$ & $191(69)$ & $<0.001$ \\
\hline Aminoglycosides, $n(\%)$ & 0 & $41(51)$ & $79(28)$ & $<0.001$ \\
\hline Anti-Gram-positive agents, $n(\%)$ & 0 & $20(30)$ & $30(11)$ & $<0.01$ \\
\hline Antifungal agents, $n(\%)$ & 0 & $20(25)$ & $33(12)$ & $<0.01$ \\
\hline Azoles, $n(\%)$ & 0 & $18(22)$ & $28(10)$ & $<0.01$ \\
\hline Echinocandins, $n(\%)$ & 0 & $1(1)$ & $4(1)$ & NS \\
\hline Duration of AIT, days, median [IQR] & $3 / 8$ & $7[3-15]$ & $4[1-10]$ & $<0.01$ \\
\hline ICU length of stay, days, median [IQR] & 0 & $14[4-27]$ & 8 [3-19] & $<0.05$ \\
\hline ICU readmission, $n(\%)$ & $0 / 1$ & $5(6)$ & $22(8)$ & NS \\
\hline Hospital mortality, n (\%) & 0 & $22(27)$ & $71(26)$ & NS \\
\hline Time to death, days, median [IQR] & 0 & $31[7-43]$ & $3[1-19]$ & $<0.01$ \\
\hline
\end{tabular}

NS non-significant

${ }^{*}$ According to the definition of the SOFA score 
Table 4 Clinical features of patients receiving AIT on admission or delayed AIT

\begin{tabular}{|c|c|c|c|c|}
\hline & Missing data & $\begin{array}{l}\text { Patients receiving } \\
\text { AlT from day } 0 n=340\end{array}$ & $\begin{array}{l}\text { Patients receiving AIT between } \\
\text { day }>0 \text { and day } 30 n=226\end{array}$ & $p$ value \\
\hline Male, $n(\%)$ & $0 / 4$ & $227(68)$ & $147(65)$ & NS \\
\hline Age, years, median [IQR] & $1 / 1$ & 59 [49-73] & $58[47-70]$ & NS \\
\hline \multicolumn{5}{|l|}{ Clinical presentation on day 0} \\
\hline Delay pain/ICU admission, days, median [IQR] & 0 & $4[1-11]$ & $2[1-5]$ & $<0.001$ \\
\hline Oliguria/anuria, $n(\%)$ & 0 & $151(44)$ & $99(44)$ & NS \\
\hline BISAP score, median [IQR] & $4 / 14$ & $2[1-2]$ & $1[0-2]$ & $<0.001$ \\
\hline Balthazar score grade $\mathrm{E}, n(\%)$ & 0 & $196(58)$ & $155(69)$ & $<0.05$ \\
\hline \multicolumn{5}{|l|}{ Severity criteria on day 0} \\
\hline SOFA score, median [IQR] & $1 / 11$ & $5[3-7]$ & $4[3-6]$ & $<0.05$ \\
\hline Respiratory failure*, $n(\%)$ & 0 & $129(38)$ & $68(30)$ & NS \\
\hline Cardiovascular failure*, n (\%) & 0 & $157(46)$ & $40(18)$ & $<0.001$ \\
\hline Renal failure*, $n(\%)$ & 0 & $65(19)$ & $51(23)$ & NS \\
\hline Septic shock, $n(\%)$ & 0 & $106(31)$ & $4(2)$ & $<0.001$ \\
\hline Acute respiratory distress syndrome, $n(\%)$ & 0 & $36(11)$ & $16(7)$ & NS \\
\hline \multicolumn{5}{|l|}{ Therapeutic management on day 0} \\
\hline Vasoactive support, $n$ (\%) & $0 / 1$ & $160(47)$ & $42(19)$ & $<0.001$ \\
\hline Mechanical ventilation, $n$ (\%) & $0 / 4$ & $126(38)$ & $57(25)$ & $<0.01$ \\
\hline Fluid loading, $n(\%)$ & $1 / 9$ & $270(82)$ & $169(75)$ & NS \\
\hline Renal replacement therapy, $n(\%)$ & $1 / 7$ & $40(12)$ & $20(9)$ & NS \\
\hline \multicolumn{5}{|l|}{ Main treatments between day $>0$ and day 30} \\
\hline Need for red blood cells transfusion, $n(\%)$ & 0 & $113(33)$ & $78(35)$ & NS \\
\hline Duration of mechanical ventilation, days, median [IQR] & $2 / 8$ & $3[0-11]$ & $9[0-21]$ & $<0.001$ \\
\hline Vasoactive support, $n$ (\%) & $0 / 1$ & $205(60)$ & $126(56)$ & NS \\
\hline Renal replacement therapy, n (\%) & $1 / 0$ & $91(27)$ & $85(38)$ & $<0.01$ \\
\hline Duration of RRT, days, median [IQR] & $5 / 6$ & $5[2-15]$ & $10[3-16]$ & NS \\
\hline Duration of AIT, days, median [IQR] & $13 / 11$ & $5[2-12]$ & $7[3-14]$ & $<0.01$ \\
\hline \multicolumn{5}{|l|}{ Main complications between day >0 and day 30} \\
\hline Acute respiratory distress syndrome, $n(\%)$ & $0 / 2$ & $100(30)$ & $101(45)$ & $<0.001$ \\
\hline Septic shock, $n(\%)$ & $16 / 30$ & $123(40)$ & $85(40)$ & NS \\
\hline Pancreatic necrosis, $n(\%)$ & $2 / 16$ & $211(65)$ & $174(78)$ & $<0.01$ \\
\hline Infected necrosis, n (\%) & $6 / 22$ & $113(36)$ & $83(38)$ & NS \\
\hline Gastro-intestinal perforation, $n(\%)$ & $9 / 21$ & $28(9)$ & $22(10)$ & NS \\
\hline Vascular thrombosis, $n(\%)$ & $5 / 20$ & $50(16)$ & $33(15)$ & NS \\
\hline Acute mesenteric ischaemia, $n(\%)$ & $7 / 20$ & $35(11)$ & $27(12)$ & NS \\
\hline Intra-abdominal collection, $n(\%)$ & $5 / 16$ & $147(45)$ & $89(40)$ & NS \\
\hline Abdominal compartment syndrome, $n(\%)$ & $4 / 21$ & $30(9)$ & $35(16)$ & $<0.05$ \\
\hline Haemorrhage, $n(\%)$ & $6 / 20$ & $46(14)$ & $33(15)$ & NS \\
\hline Peritonitis, $n(\%)$ & $8 / 22$ & $58(18)$ & $25(11)$ & $<0.05$ \\
\hline Cholangitis, $n(\%)$ & $7 / 18$ & $41(13)$ & $16(7)$ & $<0.05$ \\
\hline Digestive fistula, $n(\%)$ & $8 / 20$ & $21(7)$ & $9(4)$ & NS \\
\hline \multicolumn{5}{|l|}{ Clinical management between day $>0$ and day 30} \\
\hline Endoscopic necrosectomy, $n(\%)$ & $2 / 4$ & $83(25)$ & $64(29)$ & NS \\
\hline Surgical necrosectomy, n (\%) & $2 / 4$ & $131(39)$ & $86(38)$ & NS \\
\hline
\end{tabular}


Table 4 Clinical features of patients receiving AIT on admission or delayed AIT (Continued)

\begin{tabular}{|c|c|c|c|c|}
\hline & Missing data & $\begin{array}{l}\text { Patients receiving } \\
\text { AIT from day } 0 n=340\end{array}$ & $\begin{array}{l}\text { Patients receiving AIT between } \\
\text { day }>0 \text { and day } 30 n=226\end{array}$ & $p$ value \\
\hline Radiological drainage, $n(\%)$ & $2 / 4$ & $72(21)$ & $47(21)$ & NS \\
\hline Duration of ICU stay, days, median [IQR] & 0 & 8 [3-22] & $17[7-34]$ & $<0.001$ \\
\hline ICU readmission, $n(\%)$ & $0 / 1$ & $27(8)$ & $23(10)$ & NS \\
\hline Hospital mortality rate, $n(\%)$ & 0 & $91(27)$ & $50(22)$ & NS \\
\hline Time to death, days, median [IQR] & 0 & $8[1-31]$ & $17[5-38]$ & $<0.05$ \\
\hline
\end{tabular}

NS non-significant, ERCP endoscopic retrograde cholangiopancreatography

*According to the definition of the SOFA score

drivers of hospital mortality rather than infection and AIT.

In our study population, heterogeneity of practice is illustrated by major between-centre variability in terms of the incidence of infections, management of AIT and therapeutic interventions and prognosis, highlighting the need to more clearly define the indications for and modalities of AIT in AP patients and to analyse the results of treatment very carefully.

Recent guidelines do not recommend $\mathrm{AB}$ prophylaxis for prevention of infection of pancreatic necrosis [2]. $A B$ prophylaxis has been extensively described in multicentre studies. In the EPIC-II study reporting the prevalence of infection in ICU patients, de Waele et al. observed prophylaxis in $24 \%$ of $A P$ patients receiving $A B$ [9]. In an Indian multicentre study comprising $24 \%$ of ICU cases, $67 \%$ of patients received $\mathrm{AB}$, including $\mathrm{AB}$ prophylaxis in two thirds of cases [16]. Interestingly, prophylaxis was not reported in our cohort, which could be at least partially related to the 2001 French consensus recommendations discouraging its use in AP [17]. More recently, several studies $[18,19]$ and meta-analyses $[20$, 21] did not demonstrate any benefit for prophylaxis, which might also have influenced the prescribers' decisions. Consequently, our data provide an interesting opportunity to assess the impact of AIT with curative intent on the outcome of ICU cases of AP.

The proportion of patients receiving AIT at the time of ICU admission was similar to that reported in the EPIC-II trial, in which $45 \%$ of patients received antibiotic therapy during the first week after admission to the ICU [9]. In a recent British national review analysing $A B$ use for AP at the hospital level, AB therapy was administered to $62 \%$ of patients, while second and third courses were reported in 41 and $24 \%$ of cases, respectively [22]. The proportion of cases treated for intra-abdominal infection on ICU admission was much higher in our cohort than in the EPIC cohort (less than 30\% in the first week of ICU stay) and the frequency of pneumonia on ICU admission in our cohort was similar to the rate reported by de Waele et al. (44/116 (28\%) cases) [9]. In another large cohort of 173 infected patients with AP, Besselink et al. reported 98 (57\%) cases of infected necrosis and 84 (49\%) cases of pneumonia, but these rates were reported for the entire stay [23]. In another retrospective study focusing on extrapancreatic complications in ICU patients with AP, infectious complications were observed in 56/103 (54\%) patients with a predominance of respiratory and urinary tract infections (43\% and $21.5 \%$ of all infectious complications, respectively) [6].

The large use of carbapenems in our cohort is not surprising. The broad spectrum of these drugs and their diffusion in pancreatic tissue could at least partially explain the choice of these agents [24]. Additional explanations for these findings could be related to case-mix, local epidemiology, and surgical and endoscopic practices. In a British survey, carbapenems were the agents most commonly used in patients receiving a second course of antibiotics [22]. In line with the guidelines of the Surviving Sepsis Campaign, the use of carbapenems could be considered to be a marker of severity [15]. The selection pressure related to the extensive use of carbapenems has been previously reported to be a risk factor for the emergence of multidrug resistant organisms in many conditions $[25,26]$. In the current context of dissemination of multidrug resistant Gram-negative bacilli, cautious use of carbapenems could be proposed in many cases. However, local epidemiology remains a key issue in this setting.

A limited number of AF treatments were administered both on admission and during the ICU stay. De Waele et al. reported low proportions of fungal infections [9], while other authors have suggested a growing role of fungal infections in AP. However, the patient profiles reported in the literature are quite different, marked by prolonged antibiotic therapy and ICU stay $[27,28]$, conditions rarely observed in our patients. Most of our cases received azoles in the context of both documented and empirical AF therapy, which could be explained by the fact that these data were collected several years ago and the proportion of echinocandins may have increased over recent years. However, the diffusion of echinocandins into necrotic pancreatic tissues needs to be formally demonstrated. 
Table 5 Clinical features analysed for the patients receiving carbapenem or other AIT between day $>0$ and day 30

\begin{tabular}{|c|c|c|c|c|}
\hline & Missing data & Carbapenems $n=202$ & Other AIT $n=364$ & $p$ value \\
\hline \multicolumn{5}{|l|}{ Severity criteria on admission } \\
\hline SOFA score, median [IQR] & $4 / 8$ & $5[3-7]$ & $5[3-6]$ & NS \\
\hline Respiratory failure*, $n(\%)$ & 0 & $74(37)$ & $123(34)$ & NS \\
\hline Cardiovascular failure*,$n(\%)$ & 0 & $79(39)$ & $118(32)$ & NS \\
\hline Renal failure*,$n(\%)$ & 0 & $39(19)$ & $77(21)$ & NS \\
\hline Septic shock, $n(\%)$ & 0 & $45(22)$ & $65(18)$ & NS \\
\hline Acute respiratory distress syndrome, $n(\%)$ & 0 & $27(13)$ & $25(7)$ & $<0.05$ \\
\hline \multicolumn{5}{|l|}{ Main treatments between day $>0$ and day 30} \\
\hline Need for red blood cell transfusion, $n(\%)$ & 0 & $87(43)$ & $104(29)$ & $<0.001$ \\
\hline Number of days of mechanical ventilation & $5 / 5$ & $12[2-26]$ & $2[0-10]$ & $<0.001$ \\
\hline Vasoactive support, $n$ (\%) & $0 / 1$ & $147(73)$ & $184(51)$ & $<0.001$ \\
\hline Renal replacement therapy, $n$ (\%) & $0 / 1$ & $82(41)$ & $94(26)$ & $<0.001$ \\
\hline Duration of RRT, days, median [IQR] & $6 / 5$ & $10[3-22]$ & $5[2-13]$ & $<0.01$ \\
\hline \multicolumn{5}{|l|}{ Main complications between day $>0$ and day 30} \\
\hline Acute respiratory distress syndrome, $n(\%)$ & $1 / 1$ & $101(50)$ & $100(28)$ & $<0.001$ \\
\hline Septic shock, $n(\%)$ & $15 / 31$ & $102(55)$ & $106(32)$ & $<0.001$ \\
\hline Pancreatic necrosis, $n$ (\%) & $4 / 14$ & $156(79)$ & $229(65)$ & $<0.001$ \\
\hline Infected necrosis, $n(\%)$ & $8 / 20$ & $96(49)$ & $100(29)$ & $<0.001$ \\
\hline Gastro-intestinal perforation, $n(\%)$ & $12 / 18$ & $23(12)$ & $27(8)$ & NS \\
\hline Vascular thrombosis, $n(\%)$ & $9 / 16$ & $36(19)$ & $47(14)$ & NS \\
\hline Acute mesenteric ischaemia, $n(\%)$ & 10/17 & $26(14)$ & $36(10)$ & NS \\
\hline Intra-abdominal collection, $n(\%)$ & $6 / 15$ & $105(54)$ & $131(38)$ & $<0.001$ \\
\hline Abdominal compartment syndrome, $n(\%)$ & $9 / 17$ & $33(17)$ & $32(9)$ & $<0.01$ \\
\hline Haemorrhage, $n(\%)$ & $8 / 18$ & $44(23)$ & $35(10)$ & $<0.001$ \\
\hline Peritonitis, $n(\%)$ & $11 / 19$ & $36(19)$ & $47(14)$ & NS \\
\hline Cholangitis, n (\%) & $8 / 17$ & $18(9)$ & $39(11)$ & NS \\
\hline Digestive fistula, $n(\%)$ & 10/18 & $11(6)$ & $19(5)$ & NS \\
\hline \multicolumn{5}{|c|}{ Clinical management between day $>0$ and day 30} \\
\hline Endoscopic necrosectomy, n (\%) & $1 / 5$ & $76(38)$ & $71(20)$ & $<0.001$ \\
\hline Surgical necrosectomy, n (\%) & $1 / 5$ & $94(47)$ & $123(34)$ & $<0.01$ \\
\hline Radiological drainage, $n(\%)$ & $1 / 5$ & $63(31)$ & $56(16)$ & $<0.001$ \\
\hline \multicolumn{5}{|c|}{ Main reasons for anti-infective therapy between day $>0$ and day 30} \\
\hline Intra-abdominal infection, $n(\%)$ & $11 / 13$ & $153(80)$ & $194(55)$ & $<0.001$ \\
\hline Pneumonia, $n(\%)$ & $11 / 14$ & $79(41)$ & $89(25)$ & $<0.001$ \\
\hline Bacteraemia, $n(\%)$ & $10 / 15$ & $60(31)$ & $76(22)$ & $<0.05$ \\
\hline \multicolumn{5}{|c|}{ Anti-infective therapy administered between day $>0$ and day 30} \\
\hline Duration of AIT, days, median [IQR] & $8 / 16$ & $10[3-17]$ & $5[2-10]$ & $<0.001$ \\
\hline Aminoglycosides, $n(\%)$ & $4 / 19$ & $129(65)$ & $118(34)$ & $<0.001$ \\
\hline Anti-Gram-positive agents, $n$ (\%) & $10 / 23$ & $101(53)$ & $60(18)$ & $<0.001$ \\
\hline Antifungal agents, $n(\%)$ & $0 / 2$ & $54(27)$ & $56(15)$ & $<0.01$ \\
\hline Azoles, $n(\%)$ & $6 / 2$ & $32(16)$ & $35(10)$ & $<0.05$ \\
\hline Echinocandins, $n$ (\%) & $6 / 2$ & $24(12)$ & $20(6)$ & $<0.01$ \\
\hline Duration of ICU stay, days, median [IQR] & 0 & 22 [9-39] & $8[3-19]$ & $<0.001$ \\
\hline ICU readmission $n(\%)$ & $1 / 0$ & $21(10)$ & $29(8)$ & NS \\
\hline
\end{tabular}


Table 5 Clinical features analysed for the patients receiving carbapenem or other AIT between day >0 and day 30 (Continued)

\begin{tabular}{lllll}
\hline & Missing data & Carbapenems $n=202$ & Other AIT $n=364 \quad$ & $77(21)$ \\
\hline Hospital mortality rate, $n(\%)$ & 0 & $64(32)$ & $29[11-45]$ & $4[1-18]$ \\
Time to death, days, median $[\mathrm{IQR}]$ & 0 & 29.01 & $<0.001$
\end{tabular}

NS non-significant, NA not applicable, ERCP endoscopic retrograde cholangiopancreatography

*According to the definition of the SOFA score

The mortality rate in our cohort was similar to that previously reported $[6,8,9,23]$. Patients who received delayed AIT had poorer outcomes in terms of morbidity, nosocomial infections and length of ICU stay, despite a less severe clinical status on admission. Interestingly, AIT-free patients had similar mortality rates to those receiving AIT related to non-infectious complications. The role of infection and AIT on prognosis was not demonstrated in multivariate analysis, despite forcing these criteria into the analysis, suggesting that initial severity plays a major role.

Our study has several limitations. The retrospective nature of the study is obviously an important issue. However, prospective registries including such a large number of cases would appear to be difficult to achieve, and to the best of our knowledge, no such studies have yet been published. The lack of information on AIT before ICU admission is another important limitation to understand the prescribers' treatment decisions. The indications for AIT were left to the discretion of the attending physicians, and between-centre variability is obviously a key point. The prescriber's choices are based on microbiologically proven or suspected sites of infection motivated by the recommendations of microbiology laboratories. In a previous paper, we have reported a similar decision-making process for initiation and management of AIT in French ICUs [29]. The high betweencentre variability in terms of mortality rates is another illustration of the specific case-mix admitted to ICUs. This variability led us to adjust our multivariate analysis to take this factor into account. The adequacy of $A B$ therapy and the pharmacokinetic issues in these severely ill patients also need to be evaluated. The complete microbiological details were not available in our cohort,

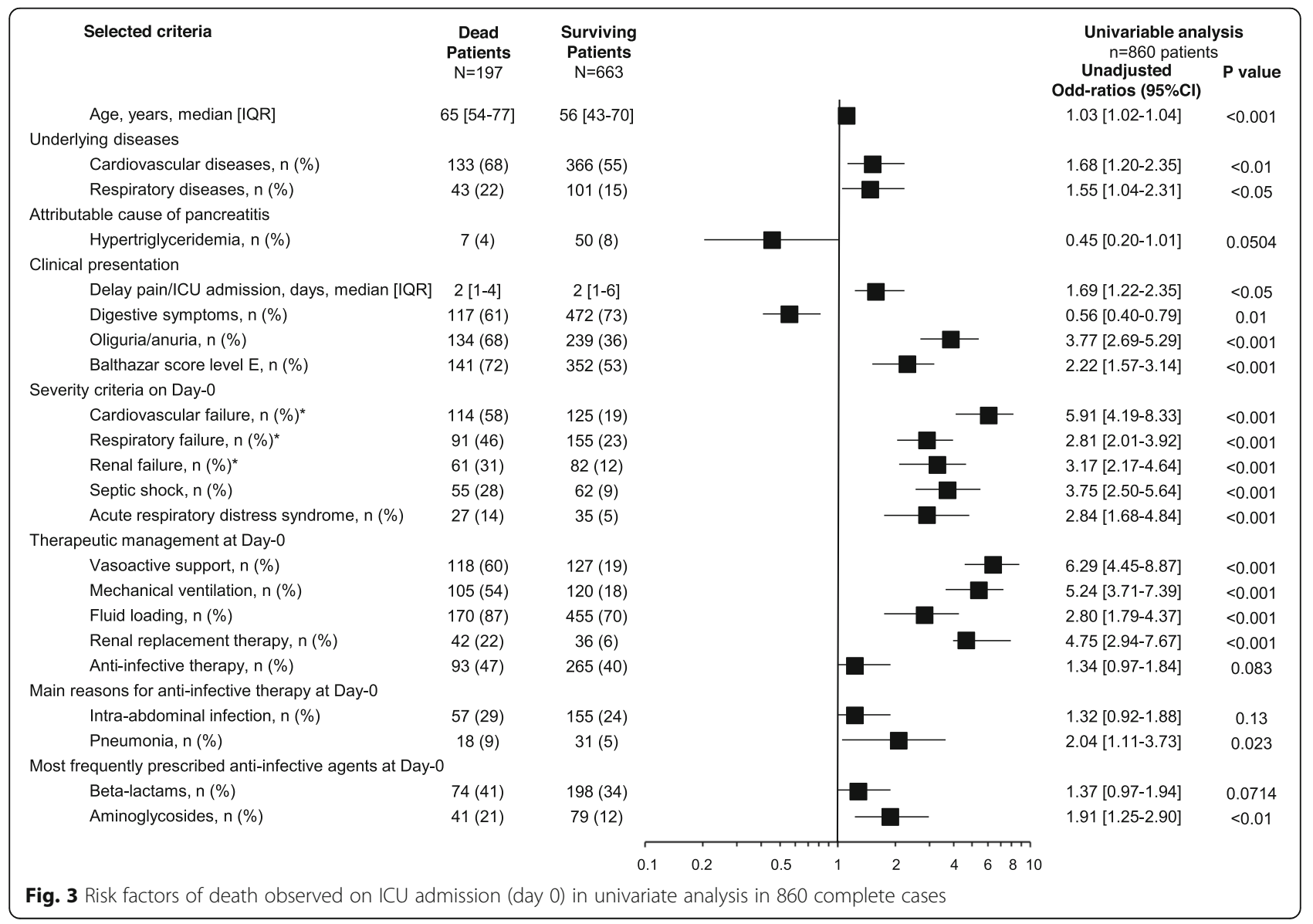




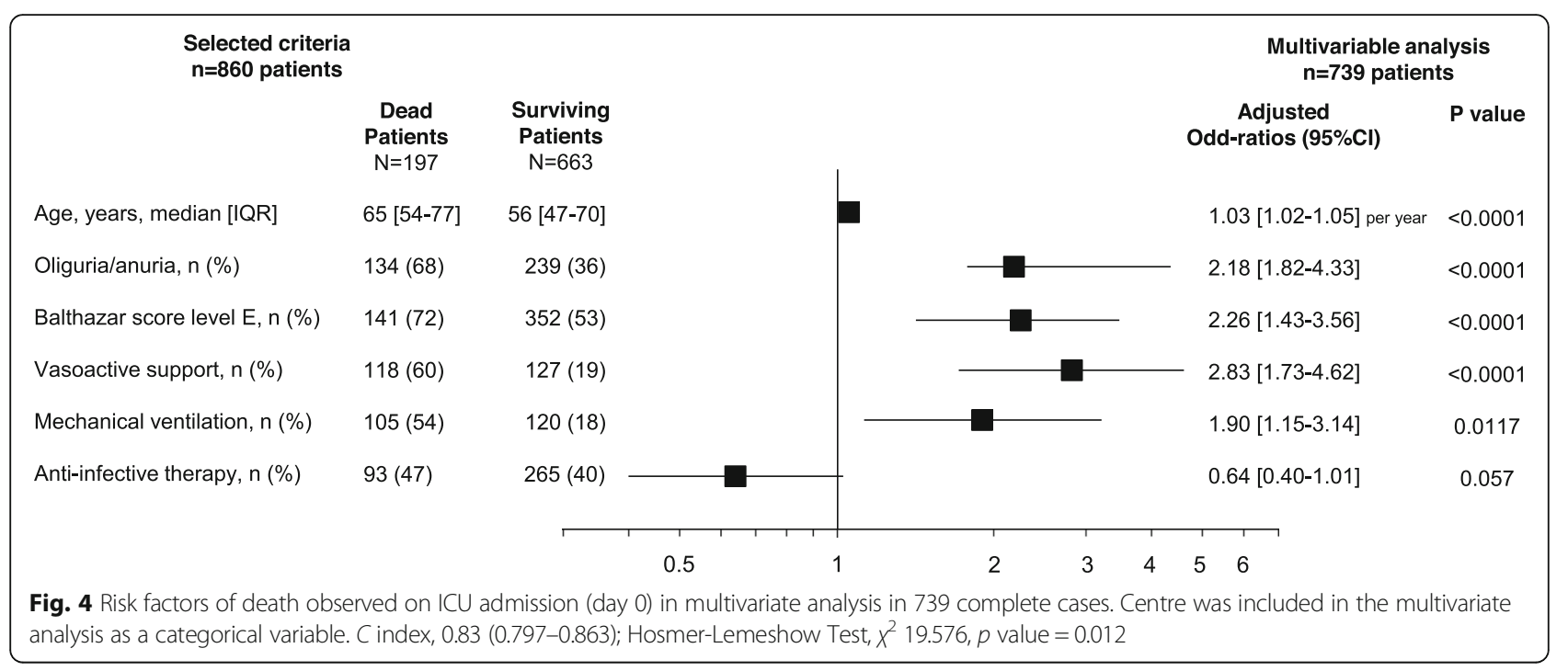

and the role played by various microorganisms, such as Enterobacteriaceae, enterococci or yeasts, needs to be analysed in more detail. The 30-day timeframe of our analysis provides an incomplete overview of the use of AIT in these cases. This time interval may be too short to demonstrate the emergence of certain specific effects, such as fungal nosocomial infections reported by some authors after prolonged AIT or prolonged ICU stay [27]. Finally, the evaluation at hospital discharge is also a limitation, as the short hospital stay in several cases could limit the validity of our findings, as some late-onset complications may have been missed.

\section{Conclusion}

This multicentre, retrospective analysis illustrates the challenges faced by intensivists in the management of patients admitted for AP. High proportions of these patients receive AIT, both on admission and during their ICU stay, mainly for intra-abdominal and pulmonary infections. Mixed sources of infection are additional indications of AIT during the ICU stay. A large heterogeneity is observed between centres in terms of incidence of infections, AIT prescribing practices, therapeutic management and outcome. Overall, AIT reflects the initial severity and complications of AP, but is not a risk factor for death.

\section{Supplementary information}

Supplementary information accompanies this paper at https://doi.org/10. 1186/s13054-019-2681-5.

Additional file 1: Figure S1. Proportions (expressed per centre) of patients treated for septic shock (panel A) or abdominal sepsis (B) and/or pneumonia (C) on Day-0 and between Day $>0$ and Day 30. (data not available for centre G). Figure S2. Proportions (expressed per centre) of patients receiving antibiotic agents (panel $\mathrm{A}$ ), and antifungal agents (B) on Day-0 and between Day $>0$ and Day-30. Figure S3. Proportions (expressed per centre) of patients receiving carbapenems (panel A), aminoglycosides (B), and anti-Gram-positive agents (C) on Day-0 and between Day $>0$ and Day-30. Figure S5. Mortality rates (expressed per centre).

Additional file 2: Figure S4. Proportions (expressed per centre) of patients who underwent endoscopic (panel A), surgical (B), percutaneous (C) or no therapeutic intervention (D) between Day>0 and Day30.

Additional file 3: Table S1. Clinical features for the patients receiving AIT on Day-0 according to empirical or documented prescription. Table S2. Comparison of the clinical features of the patients admitted in the three ICUs with the lowest mortality rates $(<10 \%)$ and the three ICUs with the highest mortality rates (>35\%)

\section{Acknowledgements}

The authors thank Dr. Bruno Pereira for his review and his comments concerning the statistical analysis.

Authors' contributions

PM, EK, MJ, JMC and HD made substantial contributions to the study concept and design, acquisition of data, analysis and interpretation of data, first drafting of the manuscript and critical revision of the manuscript for important intellectual content. All the other authors made substantial contributions to the acquisition of data and critical revision of the manuscript for important intellectual content. All authors read and approved the final manuscript.

\section{Funding}

None.

\section{Availability of data and materials}

The datasets generated during and/or analysed during the current study are available from the corresponding author on reasonable request.

Ethics approval and consent to participate

This study was approved by the French Society of Anaesthesiology and Critical Care Medicine Ethics Committee (00010254-2015-017) and the French Personal Data Protection Agency (16-023).

Consent for publication

According to French legislation, this observational study did not require the patients' informed consent.

Competing interests

The authors declare that they have no competing interests. 


\section{Author details}

'Département d'Anesthésie-Réanimation, CHU Bichat-Claude Bernard, HUPNVS, APHP, 48 rue Henri Huchard, F-75018 Paris, France. ${ }^{2}$ Université de Paris, Paris, France. ${ }^{3}$ INSERM UMR 1152 - Université de Paris, Paris, France. ${ }^{4}$ Département de Médecine Post-opératoire, CHU Clermont-Ferrand, Clermont-Ferrand, France. ${ }^{5}$ Université Clermont Auvergne, CNRS UMR 6293, INSERM U1103, GReD, Clermont-Ferrand, France. ${ }^{6}$ Division of Anaesthesiology, Critical Care, Pain and Emergency Medicine, Nimes University Hospital, and EA 2992, Université Montpellier, Nîmes, France. ${ }^{7}$ Department of Anaesthesia and Critical Care, Saint-Antoine University Hospital, Assistance Publique-Hôpitaux de Paris, and Sorbonne Universités, UPMC Univ Paris 06, Paris, France. ${ }^{8}$ Surgical Intensive Care Unit, Hôpital Pontchaillou, and Inserm U 991, Université de Rennes 1, Rennes, France. ${ }^{9}$ Department of Anaesthesiology and Critical Care Medicine, Hôpital Beaujon, Assistance Publique-Hôpitaux de Paris, Paris, France. ${ }^{10}$ Medical and Surgical ICU, Amiens University Hospital and INSERM U1088, University of Picardy Jules Verne, Amiens, France.

Received: 21 July 2019 Accepted: 21 November 2019

Published online: 05 December 2019

\section{References}

1. Working Group IAP/APA Acute Pancreatitis Guidelines. IAP/APA evidencebased guidelines for the management of acute pancreatitis. Pancreatology. 2013;13(4 Suppl 2):e1-15.

2. Crockett SD, Wani S, Gardner TB, Falck-Ytter Y, Barkun AN. American Gastroenterological Association Institute guideline on initial management of acute pancreatitis. Gastroenterology. 2018;154:1096-101.

3. Baltatzis M, Mason JM, Chandrabalan V, Stathakis P, Mclntyre B, Jegatheeswaran $S$, et al. Antibiotic use in acute pancreatitis: an audit of current practice in a tertiary Centre. Pancreatology. 2016;16:946-51.

4. De Waele JJ, Vogelaers D, Hoste E, Blot S, Colardyn F. Emergence of antibiotic resistance in infected pancreatic necrosis. Arch Surg. 2004;139: $1371-5$.

5. Jain S, Mahapatra SJ, Gupta S, Shalimar, Garg PK Infected pancreatic necrosis due to multidrug-resistant organisms and persistent organ failure predict mortality in acute pancreatitis Clin Transl Gastroenterol 2018;9:190.

6. Kothari D, Struyvenberg MR, Perillo MC, Ezaz G, Freedman SD, Sheth SG. Extra-pancreatic complications, especially hemodialysis predict mortality and length of stay, in ICU patients admitted with acute pancreatitis. Gastroenterol Rep. 2018;6:202-9.

7. Moka P, Goswami P, Kapil A, Xess I, Sreenivas V, Saraya A. Impact of antibiotic-resistant bacterial and fungal infections in outcome of acute pancreatitis. Pancreas. 2018;47:489-94.

8. Pando E, Alberti P, Hidalgo J, Vidal L, Dopazo C, Caralt M, et al. The role of extra-pancreatic infections in the prediction of severity and local complications in acute pancreatitis. Pancreatology. 2018;18:486-93.

9. De Waele JJ, Rello J, Anzueto A, Moreno R, Lipman J, Sakr Y, et al. Infections and use of antibiotics in patients admitted for severe acute pancreatitis: data from the EPIC II study. Surg Infect. 2014;15:394-8.

10. Jabaudon M, Belhadj-Tahar N, Rimmele T, Joannes-Boyau O, Bulyez S, Lefrant JY, et al. Thoracic epidural analgesia and mortality in acute pancreatitis: a multicenter propensity analysis. Crit Care Med. 2018;46: e198-205.

11. Levy ML, Fink MP, Marshall JC, Abraham E, Angus D, Cook D, et al. 2001 SCCM/ESICM/ACCP/ATS/SIS International Sepsis Definitions Conference. Crit Care Med. 2003;31:1250-6.

12. Vincent JL, Moreno R, Takala J, Willatts S, De Mendonca A, Bruining H, et al. The SOFA (Sepsis-related Organ Failure Assessment) score to describe organ dysfunction/failure. On behalf of the Working Group on Sepsis-Related Problems of the European Society of Intensive Care Medicine. Intensive Care Med. 1996;22:707-10.

13. Wu BU, Johannes RS, Sun X, Tabak Y, Conwell DL, Banks PA. The early prediction of mortality in acute pancreatitis: a large population-based study. Gut. 2008;57:1698-703.

14. Societé Française d'Anesthésie-Réanimation. Antibiothérapie probabiliste des états septiques graves. Conférence d'experts. Paris: Elsevier; 2004.

15. Dellinger RP, Levy MM, Rhodes A, Annane D, Gerlach H, Opal SM, et al. Surviving sepsis campaign: international guidelines for management of severe sepsis and septic shock: 2012. Crit Care Med. 2013;41:580-637.
16. Talukdar R, Ingale P, Choudhury HP, Dhingra R, Shetty S, Joshi H, et al. Antibiotic use in acute pancreatitis: an Indian multicenter observational study. Indian J Gastroenterol. 2014;33:458-65.

17. Société Nationale Française de Gastroentérologie. Consensus conference: acute pancreatitis. Gastroenterol Clin Biol 2001;25:177-192.

18. Dellinger EP, Tellado JM, Soto NE, Ashley SW, Barie PS, Dugernier T, et al. Early antibiotic treatment for severe acute necrotizing pancreatitis: a randomized, double-blind, placebo-controlled study. Ann Surg. 2007;245: 674-83.

19. Isenmann R, Runzi M, Kron M, Kahl S, Kraus D, Jung N, et al. Prophylactic antibiotic treatment in patients with predicted severe acute pancreatitis: a placebo-controlled, double-blind trial. Gastroenterology. 2004;126:997-1004.

20. Bai Y, Gao J, Zou DW, Li ZS. Prophylactic antibiotics cannot reduce infected pancreatic necrosis and mortality in acute necrotizing pancreatitis: evidence from a meta-analysis of randomized controlled trials. Am J Gastroenterol. 2008;103:104-10.

21. Mazaki T, Ishii Y, Takayama T. Meta-analysis of prophylactic antibiotic use in acute necrotizing pancreatitis. Br J Surg. 2006;93:674-84.

22. Barrie J, Jamdar S, Smith N, McPherson SJ, Siriwardena AK, O'Reilly DA. Misuse of antibiotics in acute pancreatitis: insights from the United Kingdom's National Confidential Enquiry into patient outcome and death (NCEPOD) survey of acute pancreatitis. Pancreatology. 2018;18:721-6.

23. Besselink MG, van Santvoort HC, Boermeester MA, Nieuwenhuijs VB, van Goor H, Dejong CH, et al. Timing and impact of infections in acute pancreatitis. Br J Surg. 2009;96:267-73.

24. Wolbrink DRJ, Kolwijck E, Ten Oever J, Horvath KD, Bouwense SAW, Schouten JA. Management of infected pancreatic necrosis in the intensive care unit: a narrative review. Clin Microbiol Infect 2019 Jun 22. pii: S1198743X(19)30361-1 Epub ahead of print.

25. Maravi-Poma E, Gener J, Alvarez-Lerma F, Olaechea P, Blanco A, DominguezMunoz JE. Early antibiotic treatment (prophylaxis) of septic complications in severe acute necrotizing pancreatitis: a prospective, randomized, multicenter study comparing two regimens with imipenem-cilastatin. Intensive Care Med. 2003;29:1974-80.

26. Voor In 't Holt AF, Severin JA, Lesaffre EM, Vos MC. A systematic review and meta-analyses show that carbapenem use and medical devices are the leading risk factors for carbapenem-resistant Pseudomonas aeruginosa. Antimicrob Agents Chemother. 2014;58:2626-37.

27. Hall AM, Poole LA, Renton B, Wozniak A, Fisher M, Neal T, et al. Prediction of invasive candidal infection in critically ill patients with severe acute pancreatitis. Crit Care. 2013;17(2):R49.

28. Kochhar R, Ahammed SK, Chakrabarti A, Ray P, Sinha SK, Dutta U, et al. Prevalence and outcome of fungal infection in patients with severe acute pancreatitis. J Gastroenterol Hepatol. 2009;24:743-7.

29. Montravers P, Dupont H, Gauzit R, Veber B, Bedos JP, Lepape A. Strategies of initiation and streamlining of antibiotic therapy in 41 French intensive care units. Crit Care. 2011;15(1):R17.

\section{Publisher's Note}

Springer Nature remains neutral with regard to jurisdictional claims in published maps and institutional affiliations.

Ready to submit your research? Choose BMC and benefit from:

- fast, convenient online submission

- thorough peer review by experienced researchers in your field

- rapid publication on acceptance

- support for research data, including large and complex data types

- gold Open Access which fosters wider collaboration and increased citations

- maximum visibility for your research: over $100 \mathrm{M}$ website views per year

At BMC, research is always in progress.

Learn more biomedcentral.com/submissions 